Acta Crystallographica Section E

\section{Structure Reports}

Online

ISSN 1600-5368

Benjamin J. Coe, ${ }^{\mathrm{a} *}$ Naomi R. M. Curati, ${ }^{a}$ Simon R. Grabowski, ${ }^{a}$ Peter N. Horton ${ }^{b}$ and Michael B. Hursthouse $^{\text {b }}$

${ }^{a}$ Department of Chemistry, University of Manchester, Manchester M13 9PL, England, and

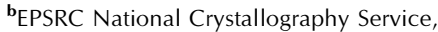
School of Chemistry, University of Southampton, Highfield, Southampton SO17 1BJ, England

Correspondence e-mail: b.coe@man.ac.uk

Key indicators

Single-crystal X-ray study

$T=120 \mathrm{~K}$

Mean $\sigma(\mathrm{C}-\mathrm{C})=0.007 \AA$

Disorder in solvent or counterion

$R$ factor $=0.060$

$w R$ factor $=0.128$

Data-to-parameter ratio $=13.1$

For details of how these key indicators were automatically derived from the article, see http://journals.iucr.org/e.

\title{
cis-Bis $\left(2,2^{\prime}\right.$-bipyridyl- $\left.\kappa^{2} N, N^{\prime}\right)$ chloro(1-phenyl- 4, $\mathbf{4}^{\prime}$-bipyridinium- $\kappa N^{1^{\prime}}$ )ruthenium(II) bis(hexa- fluorophosphate)
}

The crystal structure of the title compound, [RuCl$\left.\left(\mathrm{C}_{16} \mathrm{H}_{13} \mathrm{~N}_{2}\right)\left(\mathrm{C}_{10} \mathrm{H}_{8} \mathrm{~N}_{2}\right)_{2}\right]\left(\mathrm{PF}_{6}\right)_{2}$, is described. Although related compounds are known to display nonlinear optical (NLO) properties, the present salt crystallizes in the centrosymmetric space group $P 2_{1} / c$, so is not expected to show bulk NLO effects.

\section{Comment}

Investigations into new molecular materials having nonlinear optical (NLO) properties are important for the development of emerging optoelectronic and photonic technologies (Bosshard et al., 1995; Nalwa \& Miyata, 1997). Recent studies in this field have involved a wide range of organotransition metal complexes, which can show very pronounced NLO effects (Di Bella, 2001; Coe, 2004). Previous studies from our laboratory have included ruthenium(II) ammine complexes of $N$-arylated pyridinium ligands, such as $N$-phenyl-4,4'-bipyridinium ( $\mathrm{PhQ}^{+}$; Coe et al., 1998, 2002; Coe, Jones et al., 2003). The creation of potentially useful quadratic NLO materials requires the optimization of both molecular and macroscopic properties. Active chromophores must be arranged noncentrosymmetrically for bulk quadratic NLO effects, such as frequency doubling (second harmonic generation, $\mathrm{SHG}$ ), to be observed.

We have recently studied a series of complex salts containing cis- $\left[\mathrm{Ru}\left(\mathrm{NH}_{3}\right)_{4}(L)_{2}\right]^{4+}\left(L=\mathrm{PhQ}^{+}\right.$etc. $)$, which have strongly two-dimensional molecular NLO responses (Coe, Harris \& Brunschwig, 2003). The investigation of related compounds is clearly of interest, and readily accessible targets include species in which the ammine ligands are replaced by the classical chelating 2,2'-bipyridyl (bpy) ligand. The new compound, (I), was synthesized as an intermediate on the route to cis- $\left[\mathrm{Ru}(\mathrm{bpy})_{2}\left(\mathrm{PhQ}^{+}\right)_{2}\right]^{4+}$, by the reaction of cis$\mathrm{RuCl}_{2}(\text { bpy })_{2} \cdot 2 \mathrm{H}_{2} \mathrm{O}$ (Lay et al., 1986) with $\left[\mathrm{PhQ}^{+}\right] \mathrm{PF}_{6}($ Coe et al., 2000).

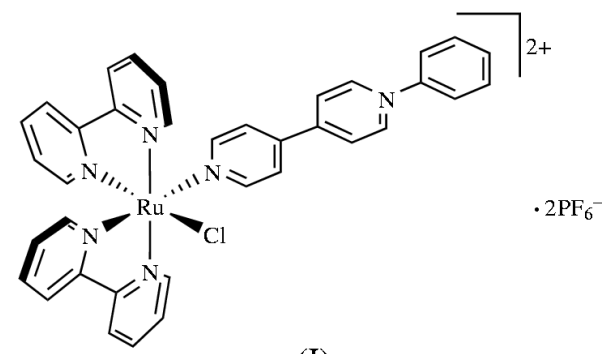

(I)

The complex salt, (I), shows an intense broad visible absorption band at $\lambda_{\max }=498 \mathrm{~nm}$ in acetonitrile. This absorption is attributable to $d \rightarrow \pi^{*}$ metal-to-ligand charge-
Received 27 August 2004 Accepted 24 September 2004 Online 9 October 2004
C 2004 International Union of Crystallography Printed in Great Britain - all rights reserved 
transfer (MLCT) transitions from the Ru-based HOMO to the LUMOs localized on the bpy and $\mathrm{PhQ}^{+}$ligands. $\mathrm{PhQ}^{+}$is expected to be a stronger electron acceptor than bpy, so it is likely that the low-energy tail of the MLCT band corresponds to $\mathrm{Ru} \rightarrow \mathrm{PhQ}^{+}$excitations. Such low-energy MLCT bands are typically associated with large molecular quadratic NLO responses (Di Bella, 2001; Coe, 2004). Cyclic voltammetric studies reveal a reversible $\mathrm{Ru}^{\mathrm{III} / \mathrm{II}}$ wave at $E_{1 / 2}=0.88 \mathrm{~V}$ versus $\mathrm{Ag}-\mathrm{AgCl}$, together with several irreversible ligand-based reduction processes, the first of which has an $E_{\mathrm{pc}}$ value of $-0.63 \mathrm{~V}$ versus $\mathrm{Ag}-\mathrm{AgCl}$ (most likely attributable to a $\mathrm{PhQ}^{+/ 0}$ process).

The molecular structure of the complex cation in (I) is as indicated by ${ }^{1} \mathrm{NMR}$ spectroscopy, with an approximately octahedral metal centre and a cis arrangement of the bpy ligands (Fig. 1). The $\mathrm{PhQ}^{+}$ligand is highly twisted, with a dihedral angle of $37.5(3)^{\circ}$ defined by the ring planes N1/C1$\mathrm{C} 5$ and $\mathrm{N} 2 / \mathrm{C} 9 / \mathrm{C} 10 / \mathrm{C} 6-\mathrm{C} 8$, and an angle of $47.0(3)^{\circ}$ between the planes N2/C9/C10/C6-C8 and C11-C16. Smaller twists between the pyridyl and pyridinium rings of $\mathrm{PhQ}^{+}$have been observed in the compounds trans- $\left[\mathrm{Ru}\left(\mathrm{NH}_{3}\right)_{4}\left(\mathrm{PTZ}\left(\mathrm{PhQ}^{+}\right)\right]\right.$$\left(\mathrm{PF}_{6}\right)_{3} \cdot \mathrm{Et}_{2} \mathrm{O}(\mathrm{PTZ}=\mathrm{S}$-coordinated phenothiazine; Coe et al., $1998)$ and trans- $\left[\mathrm{RuCl}(\mathrm{pdma})_{2}\left(\mathrm{PhQ}^{+}\right)\right]\left(\mathrm{PF}_{6}\right)_{3} \cdot \mathrm{MeCN}[\mathrm{pdma}=$ 1,2-phenylenebis(dimethylarsine); Coe et al., 2000], whilst fac$\left[\operatorname{Re}(\mathrm{CO})_{3}(L-L)\left(\mathrm{PhQ}^{+}\right)\right]\left(\mathrm{PF}_{6}\right)_{2}\left(L-L=N, N^{\prime}\right.$-bis-isopropyl-1,4diazabutadiene) also shows a highly twisted ligand structure (Busby et al., 2004).

The crystal packing of (I) is of interest with regard to quadratic NLO properties. Unfortunately, (I) adopts the centrosymmetric space group $P 2_{1} / c$ and is therefore not expected to display bulk NLO effects. Nevertheless, it is quite possible that metathesis of the hexafluorophosphate counteranions may cause the complex cations to adopt a more favourable crystal structure.

\section{Experimental}

A solution of cis- $\mathrm{RuCl}_{2}(\text { bpy })_{2} \cdot 2 \mathrm{H}_{2} \mathrm{O} \quad(100 \mathrm{mg}$. $0.192 \mathrm{mmol})$ and [ $\left.\mathrm{PhQ}^{+}\right] \mathrm{PF}_{6}(280 \mathrm{mg}, 0.740 \mathrm{mmol})$ in degassed 2:1 ethanol/acetone $(60 \mathrm{ml})$ was heated at reflux in the dark under Ar for $2.5 \mathrm{~h}$. The resulting red-purple solution was reduced in volume on a rotary evaporator, and addition of aqueous $\mathrm{NH}_{4} \mathrm{PF}_{6}$ produced a purple precipitate, which was filtered off, washed with water and dried. Excess $\left[\mathrm{PhQ}^{+}\right] \mathrm{PF}_{6}$ was removed by washing several times with methanol to afford a dark-purple solid. Yield $102 \mathrm{mg}$ (55\%). The product $(29 \mathrm{mg}$ ) was further purified by vapour diffusion of diethyl ether into a concentrated acetone solution, giving $22 \mathrm{mg}$ of darkpurple crystals. Analysis calculated for $\mathrm{C}_{36} \mathrm{H}_{29} \mathrm{ClF}_{12} \mathrm{~N}_{6} \mathrm{P}_{2} \mathrm{Ru}$ : C 44.48, $\mathrm{H}$ 3.01, N 8.65\%; found: $\mathrm{C} 44.50, \mathrm{H} 2.86, \mathrm{~N} 8.51 \%$. ${ }^{1} \mathrm{H}$ NMR (300 MHz, $\mathrm{CD}_{3} \mathrm{COCD}_{3}$, p.p.m.): $10.10\left(1 \mathrm{H}, d, J=5.7 \mathrm{~Hz}\right.$, bpy $\mathrm{H}^{6}$ ), $9.52\left(2 \mathrm{H}, d, J=7.0 \mathrm{~Hz}, \mathrm{C}_{5} \mathrm{H}_{4} \mathrm{~N}\right), 9.11\left(2 \mathrm{H}\right.$, br s, $\left.\mathrm{C}_{5} \mathrm{H}_{4} \mathrm{~N}\right), 8.80(4 \mathrm{H}, d, J=$ $\left.6.9 \mathrm{~Hz}, \mathrm{C}_{5} \mathrm{H}_{4} \mathrm{~N}\right), 8.73\left(1 \mathrm{H}, d, J=8.2 \mathrm{~Hz}\right.$, bpy $\left.\mathrm{H}^{3}\right), 8.67(1 \mathrm{H}, d, J=$ $7.8 \mathrm{~Hz}$, bpy $\left.\mathrm{H}^{3}\right), 8.64\left(1 \mathrm{H}, d, J=7.8 \mathrm{~Hz}\right.$, bpy $\left.\mathrm{H}^{3}\right), 8.26\left(2 \mathrm{H}, m\right.$, bpy $\mathrm{H}^{4}$ and $\left.\mathrm{H}^{3}\right), 8.18\left(1 \mathrm{H}, d, J=5.1 \mathrm{~Hz}\right.$, bpy $\left.\mathrm{H}^{6}\right), 8.08-7.94(7 \mathrm{H}, m, 2 \mathrm{Ph}, 3 b p y$ $\mathrm{H}^{4}$ and $\left.2 \mathrm{bpy} \mathrm{H}^{6}\right), 7.84\left(4 \mathrm{H}, m, 3 \mathrm{Ph}\right.$ and bpy $\left.\mathrm{H}^{5}\right), 7.76(1 \mathrm{H}, m, J=1.5$, 5.8 and $7.5 \mathrm{~Hz}$, bpy $\left.\mathrm{H}^{5}\right), 7.45\left(1 \mathrm{H}, m, J=1.5,5.9\right.$ and $7.1 \mathrm{~Hz}$, bpy $\left.\mathrm{H}^{5}\right)$, $7.37\left(1 \mathrm{H}, m, J=1.3,5.9\right.$ and $7.6 \mathrm{~Hz}$, bpy $\left.\mathrm{H}^{5}\right)$. ES-MS $m / z=995(\{M+$ $\left.\left.\mathrm{Na}^{+}\right\}^{+}\right), 827\left(\left\{M-\mathrm{PF}_{6}{ }^{-}\right\}^{+}\right)$. Crystals were obtained by slow diffusion of diethyl ether vapour into an acetone solution at $277 \mathrm{~K}$.

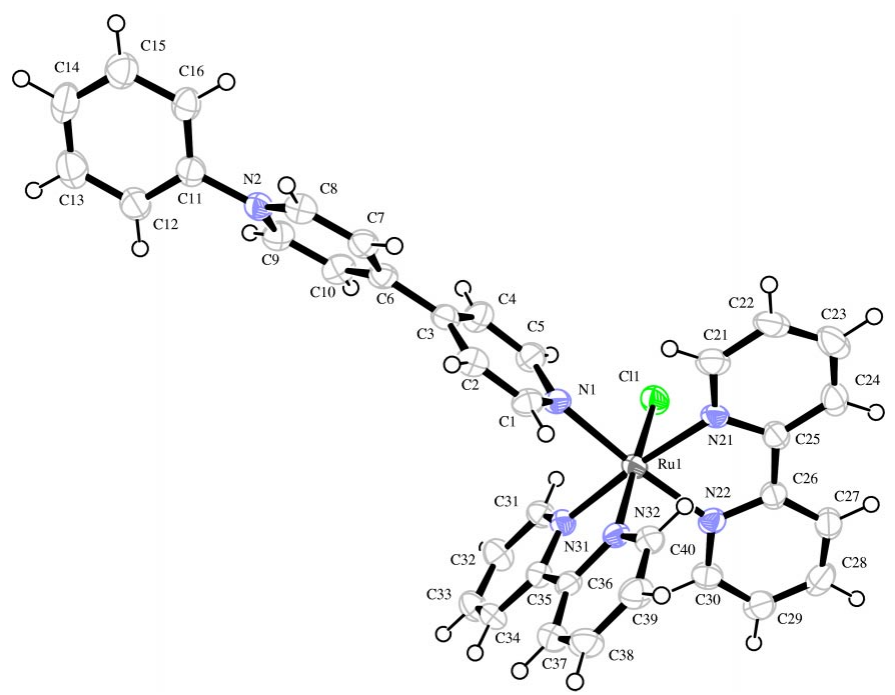

Figure 1

View of the complex cation in salt (I) (35\% probability displacement ellipsoids).

\section{Crystal data}

$\left[\mathrm{RuCl}\left(\mathrm{C}_{16} \mathrm{H}_{13} \mathrm{~N}_{2}\right)\left(\mathrm{C}_{10} \mathrm{H}_{8} \mathrm{~N}_{2}\right)_{2}\right]\left(\mathrm{PF}_{6}\right)_{2}$ $M_{r}=972.11$

Monoclinic, $P 2_{\mathrm{d}} / c$

$a=12.687(3) \mathrm{A}$ 。

$b=23.424(17) \AA$

$c=12.956(8) \AA$

$\beta=104.80(4)^{\circ}$

$V=3722(4) \AA^{3}$

$Z=4$

Data collection

Nonius KappaCCD area-detector diffractometer

$\varphi$ and $\omega$ scans

Absorption correction: multi-scan (SORTAV; Blessing, 1995, 1997)

$T_{\min }=0.783, T_{\max }=0.980$

50477 measured reflections

\section{Refinement}

Refinement on $F^{2}$

$R\left[F^{2}>2 \sigma\left(F^{2}\right)\right]=0.060$

$w R\left(F^{2}\right)=0.128$

$S=1.03$

8537 reflections

651 parameters

$\mathrm{H}$-atom parameters constrained
$D_{x}=1.735 \mathrm{Mg} \mathrm{m}^{-3}$

Mo $K \alpha$ radiation

Cell parameters from 8598

reflections

$\theta=2.9-27.5^{\circ}$

$\mu=0.68 \mathrm{~mm}^{-1}$

$T=120(2) \mathrm{K}$

Plate, dark purple

$0.38 \times 0.22 \times 0.03 \mathrm{~mm}$

8537 independent reflections 5485 reflections with $I>2 \sigma(I)$

$R_{\text {int }}=0.097$

$\theta_{\text {max }}=27.5^{\circ}$

$h=-16 \rightarrow 15$

$k=-30 \rightarrow 30$

$l=-16 \rightarrow 16$
Table 1

Selected geometric parameters $\left(\AA{ }^{\circ}{ }^{\circ}\right)$.

\begin{tabular}{lrlr}
\hline Ru1-N32 & $2.029(3)$ & $\mathrm{Ru} 1-\mathrm{N} 31$ & $2.057(3)$ \\
$\mathrm{Ru} 1-\mathrm{N} 22$ & $2.038(3)$ & $\mathrm{Ru} 1-\mathrm{N} 1$ & $2.125(3)$ \\
$\mathrm{Ru} 1-\mathrm{N} 21$ & $2.053(3)$ & $\mathrm{Ru} 1-\mathrm{Cl} 1$ & $2.4059(16)$ \\
& & & \\
$\mathrm{N} 32-\mathrm{Ru} 1-\mathrm{N} 22$ & $91.32(13)$ & $\mathrm{N} 21-\mathrm{Ru} 1-\mathrm{N} 1$ & $97.65(13)$ \\
$\mathrm{N} 32-\mathrm{Ru} 1-\mathrm{N} 21$ & $98.91(14)$ & $\mathrm{N} 31-\mathrm{Ru} 1-\mathrm{N} 1$ & $87.81(13)$ \\
$\mathrm{N} 22-\mathrm{Ru} 1-\mathrm{N} 21$ & $78.99(14)$ & $\mathrm{N} 32-\mathrm{Ru} 1-\mathrm{Cl} 1$ & $173.48(10)$ \\
$\mathrm{N} 32-\mathrm{Ru} 1-\mathrm{N} 31$ & $79.60(15)$ & $\mathrm{N} 22-\mathrm{Ru} 1-\mathrm{Cl} 1$ & $84.68(10)$ \\
$\mathrm{N} 22-\mathrm{Ru} 1-\mathrm{N} 31$ & $95.61(13)$ & $\mathrm{N} 21-\mathrm{Ru} 1-\mathrm{Cl} 1$ & $85.41(11)$ \\
$\mathrm{N} 21-\mathrm{Ru} 1-\mathrm{N} 31$ & $174.40(13)$ & $\mathrm{N} 31-\mathrm{Ru} 1-\mathrm{Cl} 1$ & $95.63(11)$ \\
$\mathrm{N} 32-\mathrm{Ru} 1-\mathrm{N} 1$ & $92.09(13)$ & $\mathrm{N} 1-\mathrm{Ru} 1-\mathrm{Cl} 1$ & $92.18(10)$ \\
$\mathrm{N} 22-\mathrm{Ru} 1-\mathrm{N} 1$ & $175.56(13)$ & &
\end{tabular}


The two hexafluorophosphate anions were both disordered over two main sites [site-occupancy factors: (i) $\mathrm{PF}_{6} 0.45$ (2):0.55 (2) P1:P101; (ii) $\mathrm{PF}_{6} 0.581$ (5):0.419 (2) P11:P111]. To simplify the modelling, the $\mathrm{P}-\mathrm{F}$ bonds were restrained to be similar in length, with angles close to $90^{\circ}$. These restraints have resulted in some closer contacts than expected. All $\mathrm{H}$ atoms were included in idealized positions, with $\mathrm{C}-\mathrm{H}=0.95 \AA$ and $U_{\text {iso }}(\mathrm{H})$ values set at $1.2 U_{\text {eq }}(\mathrm{C})$. The maximum electron-density peak is $2.23 \AA$ from atom H39.

Data collection: DENZO (Otwinowski \& Minor, 1997); cell refinement: DENZO and COLLECT (Hooft, 1998); data reduction: $D E N Z O$ and COLLECT; program(s) used to solve structure: SHELXS97 (Sheldrick, 1997); program(s) used to refine structure: SHELXL97 (Sheldrick, 1997); molecular graphics: ORTEP-3 (Farrugia, 1997); software used to prepare material for publication: Win GX (Farrugia, 1999).

The authors thank the EPSRC for funding crystallographic facilities and for a postdoctoral grant (GR/R81459).

\section{References}

Blessing, R. H. (1995). Acta Cryst. A51, 33-38.

Blessing, R. H. (1997). J. Appl. Cryst. 30, 421-426.

Bosshard, Ch., Sutter, K., Prêtre, Ph., Hulliger, J., Flörsheimer, M., Kaatz, P. \& Günter, P. (1995). Organic Nonlinear Optical Materials, Advances in Nonlinear Optics, Vol. 1. Amsterdam: Gordon and Breach.
Busby, M., Liard, D. J., Motevalli, M., Toms, H. \& Vlcek, A. Jr (2004). Inorg. Chim. Acta, 357, 167-176.

Coe, B. J. (2004). In Comprehensive Coordination Chemistry II: from Biology to Nanotechnology, Vol. 9, edited by J. A. McCleverty \& T. J. Meyer, Nonlinear Optical Properties of Metal Complexes, pp. 621-687. Oxford: Elsevier Pergamon.

Coe, B. J., Beyer, T., Jeffery, J. C., Coles, S. J., Gelbrich, T., Hursthouse, M. B. \& Light, M. E. (2000). J. Chem. Soc. Dalton Trans. pp. 797-803.

Coe, B. J., Harris, J. A. \& Brunschwig, B. S. (2002). J. Phys. Chem. A, 106, $897-$ 905.

Coe, B. J., Harris, J. A. \& Brunschwig, B. S. (2003). Dalton Trans. pp. $2384-$ 2386.

Coe, B. J., Harris, J. A., Harrington, L. J., Jeffery, J. C., Rees, L. H., Houbrechts S. \& Persoons, A. (1998). Inorg. Chem. 37, 3391-3399.

Coe, B. J., Jones, L. A., Harris, J. A., Sanderson, E. E., Brunschwig, B. S., Asselberghs, I., Clays, K. \& Persoons, A. (2003). Dalton Trans. pp. 23352341.

Di Bella, S. (2001). Chem. Soc. Rev. 30, 355-366.

Farrugia, L. J. (1997). J. Appl. Cryst. 30, 565.

Farrugia, L. J. (1999). J. Appl. Cryst. 32, 837-838.

Hooft, R. (1998). COLLECT. Nonius BV, Delft, The Netherlands.

Lay, P. A., Sargeson, A. M. \& Taube, H. (1986). Inorg. Synth. 24, 291299.

Nalwa, H. S. \& Miyata, S. (1997). Editors. Nonlinear Optics of Organic Molecules and Polymers. Boca Raton: CRC Press.

Otwinowski, Z. \& Minor, W. (1997). Methods in Enzymology, Vol. 276, Macromolecular Crystallography, Part A, edited by C. W. Carter Jr \& R. M. Sweet, pp. 307-326. New York: Academic Press.

Sheldrick, G. M. (1997). SHELXS97 and SHELXL97. University of Göttingen, Germany. 


\section{supporting information}

Acta Cryst. (2004). E60, m1562-m1564 [https://doi.org/10.1107/S1600536804024006]

cis-Bis $\left(2,2^{\prime}\right.$-bipyridyl- $\left.\kappa^{2} N, N^{\prime}\right)$ chloro(1-phenyl-4,4'-bipyridinium-

${ }_{\kappa} N^{1}$ )ruthenium(II) bis(hexafluorophosphate)

Benjamin J. Coe, Naomi R. M. Curati, Simon R. Grabowski, Peter N. Horton and Michael B.

Hursthouse

(I)

Crystal data

$\left[\mathrm{RuCl}\left(\mathrm{C}_{16} \mathrm{H}_{13} \mathrm{~N}_{2}\right)\left(\mathrm{C}_{10} \mathrm{H}_{8} \mathrm{~N}_{2}\right)_{2}\right]\left(\mathrm{PF}_{6}\right)_{2}$

$M_{r}=972.11$

Monoclinic, $P 2{ }_{1} / c$

$a=12.687(3) \AA$

$b=23.424(17) \AA$

$c=12.956(8) \AA$

$\beta=104.80(4)^{\circ}$

$V=3722(4) \AA^{3}$

$Z=4$

$F(000)=1944$

$D_{\mathrm{x}}=1.735 \mathrm{Mg} \mathrm{m}^{-3}$

Mo $K \alpha$ radiation, $\lambda=0.71073 \AA$

Cell parameters from 8598 reflections

$\theta=2.9-27.5^{\circ}$

$\mu=0.68 \mathrm{~mm}^{-1}$

$T=120 \mathrm{~K}$

Plate, red

$0.38 \times 0.22 \times 0.03 \mathrm{~mm}$

\section{Data collection}

Nonius KappaCCD area-detector diffractometer

Radiation source: Nonius FR591 rotating anode Graphite monochromator

Detector resolution: 9.091 pixels $\mathrm{mm}^{-1}$

$\varphi$ and $\omega$ scans to fill Ewald Sphere

Absorption correction: multi-scan

(SORTAV, Blessing, 1995, 1997)

$T_{\min }=0.783, T_{\max }=0.980$

50477 measured reflections

8537 independent reflections

5485 reflections with $I>2 \sigma(I)$

$R_{\text {int }}=0.097$

$\theta_{\text {max }}=27.5^{\circ}, \theta_{\text {min }}=3.1^{\circ}$

$h=-16 \rightarrow 15$

$k=-30 \rightarrow 30$

$l=-16 \rightarrow 16$

Refinement

Refinement on $F^{2}$

Least-squares matrix: full

$R\left[F^{2}>2 \sigma\left(F^{2}\right)\right]=0.060$

$w R\left(F^{2}\right)=0.128$

$S=1.03$

8537 reflections

651 parameters

612 restraints

Primary atom site location: structure-invariant direct methods

Secondary atom site location: difference Fourier map

Hydrogen site location: inferred from neighbouring sites

H-atom parameters constrained

$w=1 /\left[\sigma^{2}\left(F_{\mathrm{o}}^{2}\right)+(0.0483 P)^{2}+5.3619 P\right]$

where $P=\left(F_{\mathrm{o}}{ }^{2}+2 F_{\mathrm{c}}{ }^{2}\right) / 3$

$(\Delta / \sigma)_{\max }<0.001$

$\Delta \rho_{\max }=1.40 \mathrm{e} \AA^{-3}$

$\Delta \rho_{\min }=-0.49$ e $\AA^{-3}$ 


\section{Special details}

Geometry. All e.s.d.'s (except the e.s.d. in the dihedral angle between two 1.s. planes) are estimated using the full covariance matrix. The cell e.s.d.'s are taken into account individually in the estimation of e.s.d.'s in distances, angles and torsion angles; correlations between e.s.d.'s in cell parameters are only used when they are defined by crystal symmetry. An approximate (isotropic) treatment of cell e.s.d.'s is used for estimating e.s.d.'s involving 1.s. planes.

Refinement. Refinement of $F^{2}$ against ALL reflections. The weighted $R$-factor $w R$ and goodness of fit $S$ are based on $F^{2}$, conventional $R$-factors $R$ are based on $F$, with $F$ set to zero for negative $F^{2}$. The threshold expression of $F^{2}>\sigma\left(F^{2}\right)$ is used only for calculating $R$-factors (gt) etc. and is not relevant to the choice of reflections for refinement. $R$-factors based on $F^{2}$ are statistically about twice as large as those based on $F$, and $R$ - factors based on ALL data will be even larger.

Fractional atomic coordinates and isotropic or equivalent isotropic displacement parameters $\left(\hat{A}^{2}\right)$

\begin{tabular}{|c|c|c|c|c|c|}
\hline & $x$ & $y$ & $z$ & $U_{\text {iso }} * / U_{\text {eq }}$ & Occ. $(<1)$ \\
\hline Rul & $0.75061(3)$ & $0.111296(13)$ & $0.21958(3)$ & $0.02808(11)$ & \\
\hline $\mathrm{Cl1}$ & $0.64391(9)$ & $0.04872(4)$ & $0.08574(9)$ & $0.0369(3)$ & \\
\hline $\mathrm{C} 1$ & $0.9900(3)$ & $0.11951(17)$ & $0.2087(3)$ & $0.0333(10)$ & \\
\hline H1 & 1.0009 & 0.1338 & 0.2793 & $0.040^{*}$ & \\
\hline $\mathrm{C} 2$ & $1.0783(3)$ & $0.11734(17)$ & $0.1661(3)$ & $0.0336(10)$ & \\
\hline $\mathrm{H} 2$ & 1.1479 & 0.1298 & 0.2066 & $0.040 *$ & \\
\hline $\mathrm{C} 3$ & $1.0648(3)$ & $0.09659(17)$ & $0.0622(3)$ & $0.0308(10)$ & \\
\hline $\mathrm{C} 4$ & $0.9614(3)$ & $0.07829(19)$ & $0.0102(4)$ & $0.0373(11)$ & \\
\hline H4 & 0.9484 & 0.0632 & -0.0600 & $0.045^{*}$ & \\
\hline $\mathrm{C} 5$ & $0.8782(4)$ & $0.08157(18)$ & $0.0581(4)$ & $0.0362(10)$ & \\
\hline H5 & 0.8084 & 0.0683 & 0.0200 & $0.043^{*}$ & \\
\hline C6 & $1.1534(3)$ & $0.09743(17)$ & $0.0066(3)$ & $0.0313(10)$ & \\
\hline $\mathrm{C} 7$ & $1.2622(3)$ & $0.08703(17)$ & $0.0571(3)$ & $0.0331(10)$ & \\
\hline $\mathrm{H} 7$ & 1.2821 & 0.0765 & 0.1304 & $0.040 *$ & \\
\hline $\mathrm{C} 8$ & $1.3411(3)$ & 0.09177 (18) & 0.0027 & $0.0344(10)$ & \\
\hline H8 & 1.4152 & 0.0844 & 0.0381 & $0.041 *$ & \\
\hline C9 & $1.2097(4)$ & $0.11577(18)$ & $-0.1524(4)$ & $0.0364(10)$ & \\
\hline H9 & 1.1916 & 0.1257 & -0.2260 & $0.044^{*}$ & \\
\hline $\mathrm{C} 10$ & $1.1287(4)$ & $0.11082(19)$ & $-0.1009(3)$ & $0.0370(10)$ & \\
\hline $\mathrm{H} 10$ & 1.0549 & 0.1166 & -0.1391 & $0.044^{*}$ & \\
\hline $\mathrm{C} 11$ & $1.3976(3)$ & $0.11371(18)$ & -0.1598 & $0.0346(10)$ & \\
\hline C12 & $1.3931(4)$ & $0.1609(2)$ & $-0.2240(4)$ & $0.0431(11)$ & \\
\hline H12 & 1.3387 & 0.1893 & -0.2285 & $0.052 *$ & \\
\hline C13 & $1.4703(4)$ & $0.1655(2)$ & $-0.2816(4)$ & $0.0506(13)$ & \\
\hline H13 & 1.4696 & 0.1978 & -0.3262 & $0.061 *$ & \\
\hline $\mathrm{C} 14$ & $1.5476(4)$ & $0.1243(2)$ & $-0.2752(4)$ & 0.0493 (13) & \\
\hline H14 & 1.5998 & 0.1278 & -0.3160 & $0.059 *$ & \\
\hline $\mathrm{C} 15$ & $1.5501(4)$ & $0.0781(2)$ & $-0.2104(4)$ & $0.0458(12)$ & \\
\hline H15 & 1.6049 & 0.0499 & -0.2057 & $0.055^{*}$ & \\
\hline $\mathrm{C} 16$ & $1.4748(3)$ & 0.07181 (19) & -0.1519 (3) & $0.0357(10)$ & \\
\hline H16 & 1.4760 & 0.0395 & -0.1073 & $0.043 *$ & \\
\hline N1 & $0.8894(3)$ & $0.10280(13)$ & $0.1574(3)$ & $0.0303(8)$ & \\
\hline $\mathrm{N} 2$ & $1.3138(3)$ & $0.10693(14)$ & -0.1012 & $0.0318(8)$ & \\
\hline $\mathrm{C} 21$ & $0.8645(4)$ & $0.00676(18)$ & $0.3363(4)$ & $0.0398(11)$ & \\
\hline $\mathrm{H} 21$ & 0.9263 & 0.0183 & 0.3128 & $0.048^{*}$ & \\
\hline
\end{tabular}




\begin{tabular}{|c|c|c|c|c|c|}
\hline $\mathrm{C} 22$ & $0.8682(4)$ & $-0.04451(19)$ & $0.3905(4)$ & $0.0443(12)$ & \\
\hline $\mathrm{H} 22$ & 0.9321 & -0.0674 & 0.4040 & $0.053^{*}$ & \\
\hline $\mathrm{C} 23$ & $0.7811(5)$ & $-0.0620(2)$ & $0.4243(4)$ & $0.0486(13)$ & \\
\hline $\mathrm{H} 23$ & 0.7822 & -0.0974 & 0.4602 & $0.058^{*}$ & \\
\hline $\mathrm{C} 24$ & 0.6908 (4) & $-0.02721(19)$ & $0.4056(3)$ & $0.0411(11)$ & \\
\hline $\mathrm{H} 24$ & 0.6287 & -0.0386 & 0.4287 & $0.049^{*}$ & \\
\hline $\mathrm{C} 25$ & 0.6903 (4) & $0.02437(18)$ & $0.3530(3)$ & $0.0355(10)$ & \\
\hline $\mathrm{C} 26$ & 0.6005 (4) & $0.06549(18)$ & $0.3333(3)$ & $0.0342(10)$ & \\
\hline $\mathrm{C} 27$ & $0.5062(4)$ & $0.0586(2)$ & $0.3675(4)$ & $0.0415(11)$ & \\
\hline H27 & 0.4951 & 0.0248 & 0.4037 & $0.050^{*}$ & \\
\hline $\mathrm{C} 28$ & 0.4289 (4) & $0.1013(2)$ & $0.3483(4)$ & $0.0453(12)$ & \\
\hline $\mathrm{H} 28$ & 0.3644 & 0.0972 & 0.3721 & $0.054^{*}$ & \\
\hline $\mathrm{C} 29$ & 0.4453 (4) & $0.1496(2)$ & 0.2947 (4) & $0.0427(11)$ & \\
\hline H29 & 0.3931 & 0.1796 & 0.2815 & $0.051^{*}$ & \\
\hline $\mathrm{C} 30$ & $0.5389(3)$ & $0.15359(19)$ & $0.2607(3)$ & $0.0358(10)$ & \\
\hline H30 & 0.5498 & 0.1869 & 0.2229 & $0.043^{*}$ & \\
\hline N21 & $0.7769(3)$ & $0.04043(14)$ & $0.3160(3)$ & $0.0319(8)$ & \\
\hline N22 & $0.6160(3)$ & $0.11275(14)$ & $0.2780(3)$ & $0.0312(8)$ & \\
\hline $\mathrm{C} 31$ & $0.6522(3)$ & $0.18837(18)$ & 0.0307 (3) & $0.0340(10)$ & \\
\hline H31 & 0.6265 & 0.1541 & -0.0066 & $0.041^{*}$ & \\
\hline $\mathrm{C} 32$ & $0.6250(4)$ & $0.23930(19)$ & $-0.0205(4)$ & $0.0403(11)$ & \\
\hline H32 & 0.5789 & 0.2403 & -0.0909 & $0.048^{*}$ & \\
\hline $\mathrm{C} 33$ & $0.6650(4)$ & $0.28928(19)$ & 0.0308 (4) & $0.0397(11)$ & \\
\hline H33 & 0.6482 & 0.3251 & -0.0037 & $0.048^{*}$ & \\
\hline $\mathrm{C} 34$ & $0.7295(3)$ & $0.28589(17)$ & $0.1329(4)$ & $0.0341(10)$ & \\
\hline H34 & 0.7587 & 0.3198 & 0.1695 & $0.041^{*}$ & \\
\hline C35 & $0.7525(3)$ & $0.23367(17)$ & $0.1832(3)$ & $0.0308(9)$ & \\
\hline $\mathrm{C} 36$ & 0.8151 (3) & $0.22600(17)$ & $0.2942(3)$ & $0.0293(9)$ & \\
\hline C37 & $0.8586(4)$ & $0.27001(19)$ & 0.3628 (4) & $0.0378(11)$ & \\
\hline H37 & 0.8500 & 0.3084 & 0.3385 & $0.045^{*}$ & \\
\hline $\mathrm{C} 38$ & 0.9141 (4) & $0.25818(19)$ & $0.4658(4)$ & $0.0416(11)$ & \\
\hline H38 & 0.9450 & 0.2882 & 0.5133 & $0.050^{*}$ & \\
\hline C39 & $0.9246(4)$ & $0.2024(2)$ & $0.4997(3)$ & $0.0394(11)$ & \\
\hline H39 & 0.9629 & 0.1933 & 0.5708 & $0.047^{*}$ & \\
\hline $\mathrm{C} 40$ & 0.8789 (3) & $0.16000(18)$ & $0.4291(3)$ & $0.0343(10)$ & \\
\hline $\mathrm{H} 40$ & 0.8852 & 0.1216 & 0.4535 & $0.041^{*}$ & \\
\hline N31 & $0.7130(3)$ & $0.18468(13)$ & $0.1306(3)$ & $0.0288(8)$ & \\
\hline N32 & $0.8261(3)$ & $0.17033(14)$ & $0.3281(3)$ & $0.0281(8)$ & \\
\hline $\mathrm{F} 1$ & $1.3343(12)$ & $0.1808(5)$ & $0.4946(14)$ & $0.081(5)$ & $0.45(2)$ \\
\hline $\mathrm{F} 2$ & $1.2107(13)$ & $0.1984(7)$ & $0.5870(11)$ & $0.074(4)$ & $0.45(2)$ \\
\hline F3 & $1.1527(10)$ & $0.2741(5)$ & $0.4872(17)$ & $0.097(5)$ & $0.45(2)$ \\
\hline F4 & $1.1588(13)$ & $0.1890(8)$ & 0.4098 (12) & $0.117(6)$ & $0.45(2)$ \\
\hline F5 & $1.2749(13)$ & $0.2566(7)$ & $0.3927(18)$ & $0.124(6)$ & $0.45(2)$ \\
\hline F6 & $1.3279(8)$ & $0.2662(5)$ & $0.569(2)$ & $0.105(6)$ & $0.45(2)$ \\
\hline P1 & $1.2432(9)$ & $0.2276(4)$ & $0.4897(12)$ & $0.072(3)$ & $0.45(2)$ \\
\hline F101 & $1.3098(7)$ & $0.2751(3)$ & $0.4877(12)$ & $0.080(3)$ & $0.55(2)$ \\
\hline F102 & $1.2117(11)$ & $0.2166(7)$ & $0.5690(9)$ & $0.071(3)$ & $0.55(2)$ \\
\hline F103 & $1.1430(8)$ & $0.1748(4)$ & $0.4139(9)$ & $0.060(3)$ & $0.55(2)$ \\
\hline
\end{tabular}




\begin{tabular}{|c|c|c|c|c|c|}
\hline F104 & $1.1295(8)$ & $0.2679(5)$ & $0.4225(14)$ & $0.092(4)$ & $0.55(2)$ \\
\hline F105 & $1.2439(7)$ & $0.2296(5)$ & $0.3349(8)$ & $0.078(3)$ & $0.55(2)$ \\
\hline F106 & $1.3239(8)$ & $0.1792(4)$ & $0.4792(11)$ & $0.059(3)$ & $0.55(2)$ \\
\hline P101 & $1.2279(5)$ & $0.2245(3)$ & $0.4527(7)$ & $0.0457(16)$ & $0.55(2)$ \\
\hline F11 & $1.2027(7)$ & -0.0807 (4) & $0.2224(7)$ & $0.054(2)$ & $0.581(5)$ \\
\hline F12 & $1.0650(8)$ & $-0.0206(6)$ & $0.2242(11)$ & $0.038(3)$ & $0.581(5)$ \\
\hline F13 & $1.1163(4)$ & $-0.0208(3)$ & $0.4038(4)$ & $0.0532(17)$ & $0.581(5)$ \\
\hline F14 & $1.2366(4)$ & $0.0037(2)$ & $0.3098(5)$ & 0.0577 (18) & $0.581(5)$ \\
\hline F15 & $1.2575(4)$ & $-0.0787(2)$ & 0.4007 (3) & $0.0438(14)$ & $0.581(5)$ \\
\hline F16 & $1.0847(4)$ & $-0.10382(18)$ & $0.3157(4)$ & $0.0492(15)$ & $0.581(5)$ \\
\hline P11 & $1.1611(4)$ & $-0.0492(2)$ & $0.3136(4)$ & $0.0307(10)$ & $0.581(5)$ \\
\hline F111 & $1.1848(13)$ & $-0.0931(5)$ & $0.2491(11)$ & $0.091(6)$ & $0.419(5)$ \\
\hline F112 & $1.0578(11)$ & $-0.0255(9)$ & $0.2354(15)$ & $0.047(5)$ & $0.419(5)$ \\
\hline F113 & $1.1773(7)$ & $0.0282(3)$ & $0.3503(5)$ & $0.062(2)$ & $0.419(5)$ \\
\hline F114 & $1.2220(5)$ & $-0.0060(3)$ & $0.2088(6)$ & $0.059(2)$ & $0.419(5)$ \\
\hline F115 & $1.3033(6)$ & $-0.0400(4)$ & $0.3723(5)$ & $0.063(2)$ & $0.419(5)$ \\
\hline F116 & $1.1404(8)$ & $-0.0588(5)$ & $0.3988(7)$ & $0.078(3)$ & $0.419(5)$ \\
\hline P111 & $1.1815(6)$ & $-0.0333(3)$ & $0.3028(6)$ & $0.0393(17)$ & $0.419(5)$ \\
\hline
\end{tabular}

Atomic displacement parameters $\left(\AA^{2}\right)$

\begin{tabular}{|c|c|c|c|c|c|c|}
\hline & $U^{11}$ & $U^{22}$ & $U^{33}$ & $U^{12}$ & $U^{13}$ & $U^{23}$ \\
\hline Ru1 & $0.0348(2)$ & $0.02182(17)$ & $0.0286(2)$ & $-0.00108(15)$ & $0.00990(14)$ & $-0.00148(15)$ \\
\hline $\mathrm{Cl1}$ & $0.0439(6)$ & $0.0312(6)$ & $0.0360(6)$ & $-0.0081(5)$ & $0.0110(5)$ & $-0.0054(5)$ \\
\hline $\mathrm{C} 1$ & $0.034(2)$ & $0.035(2)$ & $0.030(2)$ & 0.0032 (19) & $0.007(2)$ & $-0.0032(19)$ \\
\hline $\mathrm{C} 2$ & $0.035(2)$ & $0.032(2)$ & $0.029(2)$ & 0.0009 (19) & 0.0006 (19) & $-0.0024(19)$ \\
\hline C3 & 0.037 (2) & $0.025(2)$ & $0.030(2)$ & $0.0036(17)$ & $0.008(2)$ & $0.0003(17)$ \\
\hline $\mathrm{C} 4$ & $0.039(3)$ & $0.044(3)$ & $0.030(2)$ & $-0.007(2)$ & $0.010(2)$ & $-0.011(2)$ \\
\hline $\mathrm{C} 5$ & $0.034(2)$ & $0.036(3)$ & $0.039(3)$ & $-0.0059(19)$ & $0.010(2)$ & $-0.007(2)$ \\
\hline C6 & 0.037 (3) & $0.027(2)$ & $0.030(2)$ & $-0.0001(17)$ & $0.008(2)$ & $-0.0063(18)$ \\
\hline $\mathrm{C} 7$ & 0.039 (3) & $0.031(2)$ & $0.030(2)$ & $-0.0003(19)$ & $0.008(2)$ & -0.0025 (19) \\
\hline $\mathrm{C} 8$ & $0.033(2)$ & $0.034(2)$ & $0.033(3)$ & 0.0007 (18) & $0.002(2)$ & $-0.0026(19)$ \\
\hline $\mathrm{C} 9$ & $0.041(3)$ & $0.039(3)$ & $0.028(2)$ & $0.000(2)$ & 0.007 (2) & $-0.005(2)$ \\
\hline $\mathrm{C} 10$ & $0.034(2)$ & $0.043(3)$ & $0.032(3)$ & $-0.001(2)$ & $0.005(2)$ & $-0.009(2)$ \\
\hline $\mathrm{C} 11$ & $0.039(2)$ & $0.033(2)$ & $0.033(2)$ & -0.009 (2) & $0.010(2)$ & $-0.008(2)$ \\
\hline $\mathrm{C} 12$ & $0.053(3)$ & $0.037(3)$ & $0.042(3)$ & $-0.005(2)$ & $0.018(2)$ & $-0.002(2)$ \\
\hline $\mathrm{C} 13$ & $0.062(3)$ & $0.047(3)$ & $0.045(3)$ & -0.010 & $0.018(3)$ & $0.004(2)$ \\
\hline C14 & $0.047(3)$ & 0.059 (3) & $0.047(3)$ & $-0.016(2)$ & $0.024(3)$ & -0.003 \\
\hline $\mathrm{C} 15$ & $0.042(3)$ & $0.048(3)$ & $0.049(3)$ & $-0.005(2)$ & $0.015(2)$ & $-0.003(2)$ \\
\hline C16 & $0.032(2)$ & $0.040(3)$ & $0.035(3)$ & $-0.007(2)$ & 0.009 (2) & $-0.003(2)$ \\
\hline N1 & $0.036(2)$ & 0.0267 (19) & $0.029(2)$ & $0.0030(15)$ & 0.0105 (16) & $-0.0019(15)$ \\
\hline N2 & $0.037(2)$ & 0.0274 (19) & $0.032(2)$ & $-0.0028(15)$ & 0.0104 (17) & $-0.0031(16)$ \\
\hline $\mathrm{C} 21$ & $0.050(3)$ & $0.032(2)$ & 0.037 (3) & $0.005(2)$ & $0.011(2)$ & $-0.006(2)$ \\
\hline $\mathrm{C} 22$ & $0.070(3)$ & $0.028(2)$ & $0.033(3)$ & $0.013(2)$ & $0.011(2)$ & $-0.003(2)$ \\
\hline $\mathrm{C} 23$ & $0.084(4)$ & $0.028(3)$ & $0.032(3)$ & $-0.004(3)$ & $0.013(3)$ & $-0.002(2)$ \\
\hline $\mathrm{C} 24$ & $0.061(3)$ & $0.037(3)$ & $0.027(3)$ & $-0.011(2)$ & $0.015(2)$ & $-0.003(2)$ \\
\hline $\mathrm{C} 25$ & $0.053(3)$ & $0.029(2)$ & $0.026(2)$ & $-0.004(2)$ & $0.013(2)$ & -0.0019 (19) \\
\hline $\mathrm{C} 26$ & $0.047(3)$ & $0.031(2)$ & $0.027(2)$ & $-0.007(2)$ & $0.013(2)$ & $-0.0050(19)$ \\
\hline
\end{tabular}




\begin{tabular}{|c|c|c|c|c|c|c|}
\hline $\mathrm{C} 27$ & $0.054(3)$ & $0.038(3)$ & $0.036(3)$ & $-0.010(2)$ & $0.018(2)$ & $-0.002(2)$ \\
\hline $\mathrm{C} 28$ & $0.044(3)$ & $0.057(3)$ & $0.040(3)$ & $-0.011(2)$ & $0.021(2)$ & $-0.011(2)$ \\
\hline $\mathrm{C} 29$ & $0.040(3)$ & $0.043(3)$ & $0.047(3)$ & $-0.002(2)$ & $0.014(2)$ & $-0.008(2)$ \\
\hline $\mathrm{C} 30$ & $0.036(3)$ & $0.033(2)$ & $0.036(3)$ & -0.0007 (19) & $0.006(2)$ & $-0.005(2)$ \\
\hline N21 & $0.050(2)$ & $0.0221(18)$ & $0.0258(19)$ & $0.0033(16)$ & $0.0131(17)$ & $-0.0030(15)$ \\
\hline $\mathrm{N} 22$ & $0.034(2)$ & $0.0311(19)$ & $0.031(2)$ & $-0.0042(16)$ & $0.0113(16)$ & $-0.0072(17)$ \\
\hline $\mathrm{C} 31$ & $0.036(2)$ & $0.031(2)$ & $0.034(3)$ & $-0.0028(18)$ & $0.007(2)$ & $-0.004(2)$ \\
\hline $\mathrm{C} 32$ & $0.043(3)$ & $0.038(3)$ & $0.035(3)$ & $-0.001(2)$ & $0.001(2)$ & $0.005(2)$ \\
\hline C33 & $0.046(3)$ & $0.032(2)$ & $0.040(3)$ & $0.004(2)$ & $0.010(2)$ & $0.010(2)$ \\
\hline C34 & $0.041(3)$ & $0.022(2)$ & $0.041(3)$ & $-0.0016(18)$ & $0.013(2)$ & 0.0003 (19) \\
\hline $\mathrm{C} 35$ & $0.031(2)$ & $0.029(2)$ & $0.035(3)$ & $-0.0016(18)$ & $0.013(2)$ & -0.0017 (19) \\
\hline $\mathrm{C} 36$ & $0.028(2)$ & $0.030(2)$ & $0.033(2)$ & 0.0002 (17) & 0.0131 (19) & $-0.0044(19)$ \\
\hline C37 & $0.044(3)$ & $0.031(2)$ & $0.039(3)$ & $-0.003(2)$ & $0.013(2)$ & $-0.003(2)$ \\
\hline C38 & $0.046(3)$ & $0.036(3)$ & $0.043(3)$ & $-0.005(2)$ & $0.012(2)$ & $-0.013(2)$ \\
\hline $\mathrm{C} 39$ & $0.046(3)$ & $0.045(3)$ & $0.026(2)$ & $0.002(2)$ & $0.008(2)$ & $-0.004(2)$ \\
\hline $\mathrm{C} 40$ & $0.042(3)$ & $0.031(2)$ & $0.031(3)$ & 0.0005 (19) & $0.013(2)$ & $-0.002(2)$ \\
\hline N31 & 0.0317 (19) & $0.0272(18)$ & $0.028(2)$ & $0.0002(14)$ & $0.0081(16)$ & $0.0010(15)$ \\
\hline N32 & 0.0308 (19) & $0.0281(18)$ & $0.027(2)$ & $-0.0017(14)$ & $0.0111(16)$ & $-0.0047(15)$ \\
\hline $\mathrm{F} 1$ & $0.112(11)$ & $0.060(9)$ & $0.064(9)$ & $0.018(7)$ & $0.009(7)$ & $-0.016(6)$ \\
\hline $\mathrm{F} 2$ & $0.104(8)$ & $0.056(8)$ & $0.064(7)$ & $0.028(6)$ & $0.021(6)$ & $0.009(5)$ \\
\hline $\mathrm{F} 3$ & $0.082(7)$ & $0.051(6)$ & $0.146(13)$ & $0.011(5)$ & $0.005(8)$ & $0.030(7)$ \\
\hline $\mathrm{F} 4$ & $0.151(12)$ & $0.115(12)$ & $0.066(8)$ & $-0.051(9)$ & $-0.005(8)$ & $0.003(8)$ \\
\hline F5 & 0.165 (13) & $0.089(11)$ & $0.134(14)$ & $-0.008(9)$ & 0.065 (12) & $0.055(11)$ \\
\hline F6 & $0.092(7)$ & $0.062(6)$ & $0.155(16)$ & -0.010 & $0.018(8)$ & $-0.049(8)$ \\
\hline $\mathrm{P} 1$ & $0.088(5)$ & $0.034(3)$ & $0.095(7)$ & -0.001 & $0.024(4)$ & $0.008(4)$ \\
\hline F101 & $0.083(5)$ & $0.048(4)$ & $0.101(9)$ & $-0.022(3)$ & 0.009 (6) & $0.002(5)$ \\
\hline F102 & $0.089(6)$ & $0.081(9)$ & $0.055(6)$ & $0.008(5)$ & $0.038(5)$ & $-0.006(5)$ \\
\hline F103 & $0.065(5)$ & $0.046(4)$ & $0.060(5)$ & -0.019 & $-0.001(4)$ & $0.009(3)$ \\
\hline F104 & $0.073(5)$ & $0.058(5)$ & $0.144(11)$ & $0.021(4)$ & $0.027(6)$ & $0.034(6)$ \\
\hline F105 & $0.087(5)$ & $0.093(7)$ & $0.057(5)$ & -0.009 (4) & $0.024(4)$ & $0.023(4)$ \\
\hline F106 & $0.054(5)$ & $0.052(7)$ & $0.080(7)$ & $0.005(4)$ & $0.032(5)$ & $-0.001(5)$ \\
\hline P101 & $0.046(2)$ & $0.031(2)$ & $0.063(4)$ & $-0.0042(15)$ & $0.018(2)$ & $0.005(2)$ \\
\hline F11 & $0.032(3)$ & $0.087(6)$ & $0.042(4)$ & $0.015(3)$ & $0.006(3)$ & $-0.017(4)$ \\
\hline F12 & $0.051(5)$ & $0.031(4)$ & $0.027(5)$ & $0.010(4)$ & 0.002 (4) & $0.003(3)$ \\
\hline F13 & $0.050(3)$ & $0.071(4)$ & $0.036(3)$ & $0.016(3)$ & $0.005(2)$ & $-0.021(3)$ \\
\hline F14 & $0.052(3)$ & 0.039 (3) & $0.079(5)$ & $-0.016(3)$ & $0.011(3)$ & $0.002(3)$ \\
\hline F15 & $0.048(3)$ & $0.043(3)$ & $0.033(3)$ & $0.011(2)$ & $-0.005(2)$ & $-0.010(2)$ \\
\hline F16 & $0.053(3)$ & $0.037(3)$ & $0.053(3)$ & $-0.008(2)$ & $0.006(2)$ & $0.009(2)$ \\
\hline P11 & $0.0355(18)$ & $0.031(2)$ & 0.0245 (19) & $0.0020(15)$ & $0.0053(13)$ & $-0.0040(15)$ \\
\hline F111 & $0.149(13)$ & $0.028(5)$ & $0.080(10)$ & $0.025(6)$ & $-0.002(7)$ & $-0.021(6)$ \\
\hline F112 & $0.040(6)$ & $0.066(10)$ & $0.036(8)$ & $-0.009(5)$ & $0.014(5)$ & $-0.008(6)$ \\
\hline F113 & $0.086(6)$ & $0.046(4)$ & $0.045(4)$ & $0.005(4)$ & $-0.003(4)$ & $-0.016(3)$ \\
\hline F114 & $0.056(5)$ & $0.079(5)$ & $0.045(5)$ & $0.007(4)$ & $0.023(4)$ & $0.020(4)$ \\
\hline F115 & $0.062(5)$ & $0.083(6)$ & $0.040(4)$ & $0.016(4)$ & $0.006(4)$ & $-0.005(4)$ \\
\hline F116 & $0.083(7)$ & $0.104(8)$ & $0.047(5)$ & $-0.028(6)$ & $0.017(5)$ & $0.027(6)$ \\
\hline P111 & $0.053(4)$ & $0.034(4)$ & $0.034(2)$ & $0.006(3)$ & $0.015(2)$ & $0.002(2)$ \\
\hline
\end{tabular}


Geometric parameters $\left(\AA,{ }^{\circ}\right)$

\begin{tabular}{|c|c|c|c|}
\hline $\mathrm{Ru} 1-\mathrm{N} 32$ & $2.029(3)$ & $\mathrm{C} 27-\mathrm{H} 27$ & 0.9500 \\
\hline $\mathrm{Ru} 1-\mathrm{N} 22$ & $2.038(3)$ & $\mathrm{C} 28-\mathrm{C} 29$ & $1.371(6)$ \\
\hline $\mathrm{Ru} 1-\mathrm{N} 21$ & $2.053(3)$ & $\mathrm{C} 28-\mathrm{H} 28$ & 0.9500 \\
\hline $\mathrm{Ru} 1-\mathrm{N} 31$ & $2.057(3)$ & $\mathrm{C} 29-\mathrm{C} 30$ & $1.372(6)$ \\
\hline Ru1-N1 & $2.125(3)$ & $\mathrm{C} 29-\mathrm{H} 29$ & 0.9500 \\
\hline $\mathrm{Ru} 1-\mathrm{Cl1}$ & $2.4059(16)$ & $\mathrm{C} 30-\mathrm{N} 22$ & $1.345(5)$ \\
\hline $\mathrm{C} 1-\mathrm{N} 1$ & $1.338(5)$ & $\mathrm{C} 30-\mathrm{H} 30$ & 0.9500 \\
\hline $\mathrm{C} 1-\mathrm{C} 2$ & $1.371(6)$ & $\mathrm{C} 31-\mathrm{N} 31$ & $1.330(5)$ \\
\hline $\mathrm{C} 1-\mathrm{H} 1$ & 0.9500 & $\mathrm{C} 31-\mathrm{C} 32$ & $1.366(6)$ \\
\hline $\mathrm{C} 2-\mathrm{C} 3$ & $1.400(6)$ & $\mathrm{C} 31-\mathrm{H} 31$ & 0.9500 \\
\hline $\mathrm{C} 2-\mathrm{H} 2$ & 0.9500 & $\mathrm{C} 32-\mathrm{C} 33$ & $1.377(6)$ \\
\hline $\mathrm{C} 3-\mathrm{C} 4$ & $1.381(6)$ & C $32-\mathrm{H} 32$ & 0.9500 \\
\hline $\mathrm{C} 3-\mathrm{C} 6$ & $1.481(6)$ & C $33-\mathrm{C} 34$ & $1.368(6)$ \\
\hline $\mathrm{C} 4-\mathrm{C} 5$ & $1.357(6)$ & C $33-\mathrm{H} 33$ & 0.9500 \\
\hline $\mathrm{C} 4-\mathrm{H} 4$ & 0.9500 & $\mathrm{C} 34-\mathrm{C} 35$ & $1.382(6)$ \\
\hline $\mathrm{C} 5-\mathrm{N} 1$ & $1.352(5)$ & C34-H34 & 0.9500 \\
\hline $\mathrm{C} 5-\mathrm{H} 5$ & 0.9500 & $\mathrm{C} 35-\mathrm{N} 31$ & $1.363(5)$ \\
\hline $\mathrm{C} 6-\mathrm{C} 10$ & $1.383(6)$ & $\mathrm{C} 35-\mathrm{C} 36$ & $1.465(6)$ \\
\hline $\mathrm{C} 6-\mathrm{C} 7$ & $1.390(6)$ & $\mathrm{C} 36-\mathrm{N} 32$ & $1.372(5)$ \\
\hline $\mathrm{C} 7-\mathrm{C} 8$ & $1.368(6)$ & $\mathrm{C} 36-\mathrm{C} 37$ & $1.381(6)$ \\
\hline $\mathrm{C} 7-\mathrm{H} 7$ & 0.9500 & C37-C38 & $1.370(6)$ \\
\hline $\mathrm{C} 8-\mathrm{N} 2$ & $1.349(5)$ & C37-H37 & 0.9500 \\
\hline $\mathrm{C} 8-\mathrm{H} 8$ & 0.9500 & $\mathrm{C} 38-\mathrm{C} 39$ & $1.373(6)$ \\
\hline $\mathrm{C} 9-\mathrm{N} 2$ & $1.334(5)$ & C $38-\mathrm{H} 38$ & 0.9500 \\
\hline $\mathrm{C} 9-\mathrm{C} 10$ & $1.367(6)$ & $\mathrm{C} 39-\mathrm{C} 40$ & $1.375(6)$ \\
\hline C9-H9 & 0.9500 & С $39-\mathrm{H} 39$ & 0.9500 \\
\hline $\mathrm{C} 10-\mathrm{H} 10$ & 0.9500 & $\mathrm{C} 40-\mathrm{N} 32$ & $1.331(5)$ \\
\hline $\mathrm{C} 11-\mathrm{C} 16$ & $1.372(6)$ & $\mathrm{C} 40-\mathrm{H} 40$ & 0.9500 \\
\hline $\mathrm{C} 11-\mathrm{C} 12$ & $1.375(6)$ & $\mathrm{F} 1-\mathrm{P} 1$ & $1.581(11)$ \\
\hline $\mathrm{C} 11-\mathrm{N} 2$ & $1.464(5)$ & $\mathrm{F} 2-\mathrm{P} 1$ & $1.579(10)$ \\
\hline $\mathrm{C} 12-\mathrm{C} 13$ & $1.380(6)$ & F3-P1 & $1.578(11)$ \\
\hline $\mathrm{C} 12-\mathrm{H} 12$ & 0.9500 & $\mathrm{~F} 4-\mathrm{P} 1$ & $1.570(11)$ \\
\hline $\mathrm{C} 13-\mathrm{C} 14$ & $1.363(7)$ & $\mathrm{F} 5-\mathrm{P} 1$ & $1.568(10)$ \\
\hline $\mathrm{C} 13-\mathrm{H} 13$ & 0.9500 & $\mathrm{~F} 6-\mathrm{P} 1$ & $1.569(10)$ \\
\hline $\mathrm{C} 14-\mathrm{C} 15$ & $1.366(7)$ & F101—P101 & $1.565(8)$ \\
\hline C14-H14 & 0.9500 & $\mathrm{~F} 102-\mathrm{P} 101$ & $1.583(8)$ \\
\hline $\mathrm{C} 15-\mathrm{C} 16$ & $1.370(6)$ & F103-P101 & $1.579(9)$ \\
\hline $\mathrm{C} 15-\mathrm{H} 15$ & 0.9500 & F104-P101 & $1.578(9)$ \\
\hline $\mathrm{C} 16-\mathrm{H} 16$ & 0.9500 & F105-P101 & $1.596(8)$ \\
\hline $\mathrm{C} 21-\mathrm{N} 21$ & $1.332(5)$ & F106-P101 & $1.586(9)$ \\
\hline $\mathrm{C} 21-\mathrm{C} 22$ & $1.386(6)$ & F11-P11 & $1.593(7)$ \\
\hline $\mathrm{C} 21-\mathrm{H} 21$ & 0.9500 & $\mathrm{~F} 12-\mathrm{P} 11$ & $1.600(8)$ \\
\hline $\mathrm{C} 22-\mathrm{C} 23$ & $1.352(7)$ & $\mathrm{F} 13-\mathrm{P} 11$ & $1.572(6)$ \\
\hline $\mathrm{C} 22-\mathrm{H} 22$ & 0.9500 & $\mathrm{~F} 14-\mathrm{P} 11$ & $1.575(5)$ \\
\hline $\mathrm{C} 23-\mathrm{C} 24$ & $1.375(7)$ & $\mathrm{F} 15-\mathrm{P} 11$ & $1.594(6)$ \\
\hline $\mathrm{C} 23-\mathrm{H} 23$ & 0.9500 & F16-P11 & $1.609(6)$ \\
\hline
\end{tabular}




\begin{tabular}{|c|c|c|c|}
\hline $\mathrm{C} 24-\mathrm{C} 25$ & $1.386(6)$ & F111—P111 & $1.570(10)$ \\
\hline $\mathrm{C} 24-\mathrm{H} 24$ & 0.9500 & F112-P111 & $1.600(11)$ \\
\hline $\mathrm{C} 25-\mathrm{N} 21$ & $1.360(5)$ & F113-P111 & $1.571(8)$ \\
\hline $\mathrm{C} 25-\mathrm{C} 26$ & $1.463(6)$ & F114-P111 & $1.575(8)$ \\
\hline $\mathrm{C} 26-\mathrm{N} 22$ & $1.360(5)$ & F115-P111 & $1.585(9)$ \\
\hline $\mathrm{C} 26-\mathrm{C} 27$ & $1.387(6)$ & F116-P111 & $1.583(8)$ \\
\hline $\mathrm{C} 27-\mathrm{C} 28$ & $1.379(7)$ & & \\
\hline N32-Ru1-N22 & $91.32(13)$ & $\mathrm{C} 25-\mathrm{N} 21-\mathrm{Ru} 1$ & $114.7(3)$ \\
\hline $\mathrm{N} 32-\mathrm{Ru} 1-\mathrm{N} 21$ & $98.91(14)$ & $\mathrm{C} 30-\mathrm{N} 22-\mathrm{C} 26$ & $118.1(4)$ \\
\hline $\mathrm{N} 22-\mathrm{Ru} 1-\mathrm{N} 21$ & 78.99 (14) & $\mathrm{C} 30-\mathrm{N} 22-\mathrm{Ru} 1$ & $126.3(3)$ \\
\hline $\mathrm{N} 32-\mathrm{Ru} 1-\mathrm{N} 31$ & $79.60(15)$ & $\mathrm{C} 26-\mathrm{N} 22-\mathrm{Ru} 1$ & $115.4(3)$ \\
\hline $\mathrm{N} 22-\mathrm{Ru} 1-\mathrm{N} 31$ & $95.61(13)$ & $\mathrm{N} 31-\mathrm{C} 31-\mathrm{C} 32$ & $122.8(4)$ \\
\hline $\mathrm{N} 21-\mathrm{Ru} 1-\mathrm{N} 31$ & $174.40(13)$ & $\mathrm{N} 31-\mathrm{C} 31-\mathrm{H} 31$ & 118.6 \\
\hline N32-Ru1-N1 & 92.09 (13) & $\mathrm{C} 32-\mathrm{C} 31-\mathrm{H} 31$ & 118.6 \\
\hline $\mathrm{N} 22-\mathrm{Ru} 1-\mathrm{N} 1$ & $175.56(13)$ & $\mathrm{C} 31-\mathrm{C} 32-\mathrm{C} 33$ & $119.5(4)$ \\
\hline $\mathrm{N} 21-\mathrm{Ru} 1-\mathrm{N} 1$ & $97.65(13)$ & $\mathrm{C} 31-\mathrm{C} 32-\mathrm{H} 32$ & 120.3 \\
\hline $\mathrm{N} 31-\mathrm{Ru} 1-\mathrm{N} 1$ & $87.81(13)$ & $\mathrm{C} 33-\mathrm{C} 32-\mathrm{H} 32$ & 120.3 \\
\hline $\mathrm{N} 32-\mathrm{Ru} 1-\mathrm{Cl} 1$ & $173.48(10)$ & $\mathrm{C} 34-\mathrm{C} 33-\mathrm{C} 32$ & $118.2(4)$ \\
\hline $\mathrm{N} 22-\mathrm{Ru} 1-\mathrm{Cl} 1$ & $84.68(10)$ & $\mathrm{C} 34-\mathrm{C} 33-\mathrm{H} 33$ & 120.9 \\
\hline $\mathrm{N} 21-\mathrm{Ru} 1-\mathrm{C} 11$ & $85.41(11)$ & $\mathrm{C} 32-\mathrm{C} 33-\mathrm{H} 33$ & 120.9 \\
\hline $\mathrm{N} 31-\mathrm{Ru} 1-\mathrm{Cl} 1$ & $95.63(11)$ & $\mathrm{C} 33-\mathrm{C} 34-\mathrm{C} 35$ & $120.6(4)$ \\
\hline N1-Ru1-Cl1 & $92.18(10)$ & $\mathrm{C} 33-\mathrm{C} 34-\mathrm{H} 34$ & 119.7 \\
\hline $\mathrm{N} 1-\mathrm{C} 1-\mathrm{C} 2$ & $124.3(4)$ & $\mathrm{C} 35-\mathrm{C} 34-\mathrm{H} 34$ & 119.7 \\
\hline $\mathrm{N} 1-\mathrm{C} 1-\mathrm{H} 1$ & 117.9 & $\mathrm{~N} 31-\mathrm{C} 35-\mathrm{C} 34$ & $120.2(4)$ \\
\hline $\mathrm{C} 2-\mathrm{C} 1-\mathrm{H} 1$ & 117.9 & $\mathrm{~N} 31-\mathrm{C} 35-\mathrm{C} 36$ & $115.2(4)$ \\
\hline $\mathrm{C} 1-\mathrm{C} 2-\mathrm{C} 3$ & $119.2(4)$ & $\mathrm{C} 34-\mathrm{C} 35-\mathrm{C} 36$ & $124.6(4)$ \\
\hline $\mathrm{C} 1-\mathrm{C} 2-\mathrm{H} 2$ & 120.4 & $\mathrm{~N} 32-\mathrm{C} 36-\mathrm{C} 37$ & $120.8(4)$ \\
\hline $\mathrm{C} 3-\mathrm{C} 2-\mathrm{H} 2$ & 120.4 & $\mathrm{~N} 32-\mathrm{C} 36-\mathrm{C} 35$ & $114.6(3)$ \\
\hline $\mathrm{C} 4-\mathrm{C} 3-\mathrm{C} 2$ & $116.4(4)$ & $\mathrm{C} 37-\mathrm{C} 36-\mathrm{C} 35$ & $124.6(4)$ \\
\hline $\mathrm{C} 4-\mathrm{C} 3-\mathrm{C} 6$ & $120.7(4)$ & $\mathrm{C} 38-\mathrm{C} 37-\mathrm{C} 36$ & $119.9(4)$ \\
\hline $\mathrm{C} 2-\mathrm{C} 3-\mathrm{C} 6$ & $122.7(4)$ & $\mathrm{C} 38-\mathrm{C} 37-\mathrm{H} 37$ & 120.1 \\
\hline $\mathrm{C} 5-\mathrm{C} 4-\mathrm{C} 3$ & $120.8(4)$ & $\mathrm{C} 36-\mathrm{C} 37-\mathrm{H} 37$ & 120.1 \\
\hline $\mathrm{C} 5-\mathrm{C} 4-\mathrm{H} 4$ & 119.6 & $\mathrm{C} 37-\mathrm{C} 38-\mathrm{C} 39$ & $119.2(4)$ \\
\hline $\mathrm{C} 3-\mathrm{C} 4-\mathrm{H} 4$ & 119.6 & $\mathrm{C} 37-\mathrm{C} 38-\mathrm{H} 38$ & 120.4 \\
\hline $\mathrm{N} 1-\mathrm{C} 5-\mathrm{C} 4$ & $123.4(4)$ & $\mathrm{C} 39-\mathrm{C} 38-\mathrm{H} 38$ & 120.4 \\
\hline $\mathrm{N} 1-\mathrm{C} 5-\mathrm{H} 5$ & 118.3 & $\mathrm{C} 38-\mathrm{C} 39-\mathrm{C} 40$ & $119.0(4)$ \\
\hline $\mathrm{C} 4-\mathrm{C} 5-\mathrm{H} 5$ & 118.3 & $\mathrm{C} 38-\mathrm{C} 39-\mathrm{H} 39$ & 120.5 \\
\hline $\mathrm{C} 10-\mathrm{C} 6-\mathrm{C} 7$ & $117.1(4)$ & $\mathrm{C} 40-\mathrm{C} 39-\mathrm{H} 39$ & 120.5 \\
\hline $\mathrm{C} 10-\mathrm{C} 6-\mathrm{C} 3$ & $119.2(4)$ & $\mathrm{N} 32-\mathrm{C} 40-\mathrm{C} 39$ & $122.9(4)$ \\
\hline $\mathrm{C} 7-\mathrm{C} 6-\mathrm{C} 3$ & $123.7(4)$ & $\mathrm{N} 32-\mathrm{C} 40-\mathrm{H} 40$ & 118.5 \\
\hline $\mathrm{C} 8-\mathrm{C} 7-\mathrm{C} 6$ & $120.8(4)$ & $\mathrm{C} 39-\mathrm{C} 40-\mathrm{H} 40$ & 118.5 \\
\hline $\mathrm{C} 8-\mathrm{C} 7-\mathrm{H} 7$ & 119.6 & $\mathrm{C} 31-\mathrm{N} 31-\mathrm{C} 35$ & $118.7(4)$ \\
\hline $\mathrm{C} 6-\mathrm{C} 7-\mathrm{H} 7$ & 119.6 & $\mathrm{C} 31-\mathrm{N} 31-\mathrm{Ru} 1$ & $126.4(3)$ \\
\hline $\mathrm{N} 2-\mathrm{C} 8-\mathrm{C} 7$ & $120.0(4)$ & $\mathrm{C} 35-\mathrm{N} 31-\mathrm{Ru} 1$ & $114.9(3)$ \\
\hline $\mathrm{N} 2-\mathrm{C} 8-\mathrm{H} 8$ & 120.0 & $\mathrm{C} 40-\mathrm{N} 32-\mathrm{C} 36$ & $118.2(3)$ \\
\hline $\mathrm{C} 7-\mathrm{C} 8-\mathrm{H} 8$ & 120.0 & $\mathrm{C} 40-\mathrm{N} 32-\mathrm{Ru} 1$ & $126.0(3)$ \\
\hline $\mathrm{N} 2-\mathrm{C} 9-\mathrm{C} 10$ & $121.1(4)$ & $\mathrm{C} 36-\mathrm{N} 32-\mathrm{Ru} 1$ & $115.8(3)$ \\
\hline
\end{tabular}




\begin{tabular}{|c|c|}
\hline $\mathrm{N} 2-\mathrm{C} 9-\mathrm{H} 9$ & 119.5 \\
\hline $\mathrm{C} 10-\mathrm{C} 9-\mathrm{H} 9$ & 119.5 \\
\hline $\mathrm{C} 9-\mathrm{C} 10-\mathrm{C} 6$ & $120.4(4)$ \\
\hline $\mathrm{C} 9-\mathrm{C} 10-\mathrm{H} 10$ & 119.8 \\
\hline $\mathrm{C} 6-\mathrm{C} 10-\mathrm{H} 10$ & 119.8 \\
\hline $\mathrm{C} 16-\mathrm{C} 11-\mathrm{C} 12$ & $122.6(4)$ \\
\hline $\mathrm{C} 16-\mathrm{C} 11-\mathrm{N} 2$ & $118.9(4)$ \\
\hline $\mathrm{C} 12-\mathrm{C} 11-\mathrm{N} 2$ & $118.5(4)$ \\
\hline $\mathrm{C} 11-\mathrm{C} 12-\mathrm{C} 13$ & $117.7(4)$ \\
\hline $\mathrm{C} 11-\mathrm{C} 12-\mathrm{H} 12$ & 121.1 \\
\hline $\mathrm{C} 13-\mathrm{C} 12-\mathrm{H} 12$ & 121.1 \\
\hline $\mathrm{C} 14-\mathrm{C} 13-\mathrm{C} 12$ & $120.7(5)$ \\
\hline $\mathrm{C} 14-\mathrm{C} 13-\mathrm{H} 13$ & 119.7 \\
\hline $\mathrm{C} 12-\mathrm{C} 13-\mathrm{H} 13$ & 119.7 \\
\hline $\mathrm{C} 13-\mathrm{C} 14-\mathrm{C} 15$ & $120.1(4)$ \\
\hline $\mathrm{C} 13-\mathrm{C} 14-\mathrm{H} 14$ & 119.9 \\
\hline $\mathrm{C} 15-\mathrm{C} 14-\mathrm{H} 14$ & 119.9 \\
\hline $\mathrm{C} 14-\mathrm{C} 15-\mathrm{C} 16$ & $121.0(5)$ \\
\hline $\mathrm{C} 14-\mathrm{C} 15-\mathrm{H} 15$ & 119.5 \\
\hline $\mathrm{C} 16-\mathrm{C} 15-\mathrm{H} 15$ & 119.5 \\
\hline $\mathrm{C} 15-\mathrm{C} 16-\mathrm{C} 11$ & $117.8(4)$ \\
\hline $\mathrm{C} 15-\mathrm{C} 16-\mathrm{H} 16$ & 121.1 \\
\hline $\mathrm{C} 11-\mathrm{C} 16-\mathrm{H} 16$ & 121.1 \\
\hline $\mathrm{C} 1-\mathrm{N} 1-\mathrm{C} 5$ & $115.8(3)$ \\
\hline $\mathrm{C} 1-\mathrm{N} 1-\mathrm{Ru} 1$ & $124.2(3)$ \\
\hline $\mathrm{C} 5-\mathrm{N} 1-\mathrm{Ru} 1$ & $119.9(3)$ \\
\hline $\mathrm{C} 9-\mathrm{N} 2-\mathrm{C} 8$ & $120.5(4)$ \\
\hline $\mathrm{C} 9-\mathrm{N} 2-\mathrm{C} 11$ & $118.8(4)$ \\
\hline $\mathrm{C} 8-\mathrm{N} 2-\mathrm{C} 11$ & $120.7(4)$ \\
\hline $\mathrm{N} 21-\mathrm{C} 21-\mathrm{C} 22$ & $122.1(4)$ \\
\hline $\mathrm{N} 21-\mathrm{C} 21-\mathrm{H} 21$ & 118.9 \\
\hline $\mathrm{C} 22-\mathrm{C} 21-\mathrm{H} 21$ & 118.9 \\
\hline $\mathrm{C} 23-\mathrm{C} 22-\mathrm{C} 21$ & $120.1(5)$ \\
\hline $\mathrm{C} 23-\mathrm{C} 22-\mathrm{H} 22$ & 120.0 \\
\hline $\mathrm{C} 21-\mathrm{C} 22-\mathrm{H} 22$ & 120.0 \\
\hline $\mathrm{C} 22-\mathrm{C} 23-\mathrm{C} 24$ & $118.5(4)$ \\
\hline $\mathrm{C} 22-\mathrm{C} 23-\mathrm{H} 23$ & 120.8 \\
\hline $\mathrm{C} 24-\mathrm{C} 23-\mathrm{H} 23$ & 120.8 \\
\hline $\mathrm{C} 23-\mathrm{C} 24-\mathrm{C} 25$ & $120.2(4)$ \\
\hline $\mathrm{C} 23-\mathrm{C} 24-\mathrm{H} 24$ & 119.9 \\
\hline $\mathrm{C} 25-\mathrm{C} 24-\mathrm{H} 24$ & 119.9 \\
\hline $\mathrm{N} 21-\mathrm{C} 25-\mathrm{C} 24$ & $120.7(4)$ \\
\hline $\mathrm{N} 21-\mathrm{C} 25-\mathrm{C} 26$ & $114.8(4)$ \\
\hline $\mathrm{C} 24-\mathrm{C} 25-\mathrm{C} 26$ & $124.5(4)$ \\
\hline $\mathrm{N} 22-\mathrm{C} 26-\mathrm{C} 27$ & $121.0(4)$ \\
\hline $\mathrm{N} 22-\mathrm{C} 26-\mathrm{C} 25$ & $114.7(4)$ \\
\hline $\mathrm{C} 27-\mathrm{C} 26-\mathrm{C} 25$ & $124.3(4)$ \\
\hline $\mathrm{C} 28-\mathrm{C} 27-\mathrm{C} 26$ & $119.4(4)$ \\
\hline
\end{tabular}

\begin{tabular}{|c|c|}
\hline $\mathrm{F} 5-\mathrm{P} 1-\mathrm{F} 6$ & $90.0(6)$ \\
\hline $\mathrm{F} 5-\mathrm{P} 1-\mathrm{F} 4$ & $89.6(7)$ \\
\hline $\mathrm{F} 6-\mathrm{P} 1-\mathrm{F} 4$ & $179.6(8)$ \\
\hline $\mathrm{F} 5-\mathrm{P} 1-\mathrm{F} 3$ & $91.0(6)$ \\
\hline $\mathrm{F} 6-\mathrm{P} 1-\mathrm{F} 3$ & $89.3(6)$ \\
\hline $\mathrm{F} 4-\mathrm{P} 1-\mathrm{F} 3$ & $90.8(6)$ \\
\hline $\mathrm{F} 5-\mathrm{P} 1-\mathrm{F} 2$ & $179.7(9)$ \\
\hline $\mathrm{F} 6-\mathrm{P} 1-\mathrm{F} 2$ & $90.2(6)$ \\
\hline $\mathrm{F} 4-\mathrm{P} 1-\mathrm{F} 2$ & $90.2(7)$ \\
\hline $\mathrm{F} 3-\mathrm{P} 1-\mathrm{F} 2$ & $88.7(6)$ \\
\hline $\mathrm{F} 5-\mathrm{P} 1-\mathrm{F} 1$ & $89.9(7)$ \\
\hline $\mathrm{F} 6-\mathrm{P} 1-\mathrm{F} 1$ & $90.1(6)$ \\
\hline $\mathrm{F} 4-\mathrm{P} 1-\mathrm{F} 1$ & $89.8(7)$ \\
\hline $\mathrm{F} 3-\mathrm{P} 1-\mathrm{F} 1$ & $178.8(8)$ \\
\hline $\mathrm{F} 2-\mathrm{P} 1-\mathrm{F} 1$ & $90.3(7)$ \\
\hline $\mathrm{F} 101-\mathrm{P} 101-\mathrm{F} 104$ & $90.6(6)$ \\
\hline $\mathrm{F} 101-\mathrm{P} 101-\mathrm{F} 103$ & $178.0(7)$ \\
\hline $\mathrm{F} 104-\mathrm{P} 101-\mathrm{F} 103$ & $88.0(5)$ \\
\hline $\mathrm{F} 101-\mathrm{P} 101-\mathrm{F} 102$ & $92.9(7)$ \\
\hline $\mathrm{F} 104-\mathrm{P} 101-\mathrm{F} 102$ & $91.4(6)$ \\
\hline $\mathrm{F} 103-\mathrm{P} 101-\mathrm{F} 102$ & $88.5(6)$ \\
\hline $\mathrm{F} 101-\mathrm{P} 101-\mathrm{F} 106$ & $91.5(5)$ \\
\hline $\mathrm{F} 104-\mathrm{P} 101-\mathrm{F} 106$ & $177.6(8)$ \\
\hline $\mathrm{F} 103-\mathrm{P} 101-\mathrm{F} 106$ & $89.9(6)$ \\
\hline $\mathrm{F} 102-\mathrm{P} 101-\mathrm{F} 106$ & $89.7(6)$ \\
\hline $\mathrm{F} 101-\mathrm{P} 101-\mathrm{F} 105$ & $89.0(5)$ \\
\hline $\mathrm{F} 104-\mathrm{P} 101-\mathrm{F} 105$ & $90.0(6)$ \\
\hline F103-P101-F105 & $89.6(6)$ \\
\hline $\mathrm{F} 102-\mathrm{P} 101-\mathrm{F} 105$ & $177.6(7)$ \\
\hline $\mathrm{F} 106-\mathrm{P} 101-\mathrm{F} 105$ & $88.8(6)$ \\
\hline F13-P11-F14 & $91.6(4)$ \\
\hline $\mathrm{F} 13-\mathrm{P} 11-\mathrm{F} 11$ & $177.1(6)$ \\
\hline $\mathrm{F} 14-\mathrm{P} 11-\mathrm{F} 11$ & $91.3(5)$ \\
\hline $\mathrm{F} 13-\mathrm{P} 11-\mathrm{F} 15$ & $90.8(4)$ \\
\hline $\mathrm{F} 14-\mathrm{P} 11-\mathrm{F} 15$ & $90.1(4)$ \\
\hline $\mathrm{F} 11-\mathrm{P} 11-\mathrm{F} 15$ & $89.2(4)$ \\
\hline $\mathrm{F} 13-\mathrm{P} 11-\mathrm{F} 12$ & $90.4(6)$ \\
\hline $\mathrm{F} 14-\mathrm{P} 11-\mathrm{F} 12$ & $90.2(6)$ \\
\hline $\mathrm{F} 11-\mathrm{P} 11-\mathrm{F} 12$ & $89.5(6)$ \\
\hline $\mathrm{F} 15-\mathrm{P} 11-\mathrm{F} 12$ & $178.7(7)$ \\
\hline $\mathrm{F} 13-\mathrm{P} 11-\mathrm{F} 16$ & $89.4(4)$ \\
\hline $\mathrm{F} 14-\mathrm{P} 11-\mathrm{F} 16$ & $178.9(5)$ \\
\hline $\mathrm{F} 11-\mathrm{P} 11-\mathrm{F} 16$ & 87.7 (4) \\
\hline $\mathrm{F} 15-\mathrm{P} 11-\mathrm{F} 16$ & $90.0(3)$ \\
\hline $\mathrm{F} 12-\mathrm{P} 11-\mathrm{F} 16$ & $89.7(6)$ \\
\hline $\mathrm{F} 111-\mathrm{P} 111-\mathrm{F} 113$ & $176.8(8)$ \\
\hline $\mathrm{F} 111-\mathrm{P} 111-\mathrm{F} 114$ & $88.2(7)$ \\
\hline F113-P111-F114 & $88.9(5)$ \\
\hline
\end{tabular}




$\begin{array}{llll}\mathrm{C} 28-\mathrm{C} 27-\mathrm{H} 27 & 120.3 & \mathrm{~F} 111-\mathrm{P} 111-\mathrm{F} 116 & 93.6(8) \\ \mathrm{C} 26-\mathrm{C} 27-\mathrm{H} 27 & 120.3 & \mathrm{~F} 113-\mathrm{P} 111-\mathrm{F} 116 & 89.3(6) \\ \mathrm{C} 29-\mathrm{C} 28-\mathrm{C} 27 & 119.8(4) & \mathrm{F} 114-\mathrm{P} 111-\mathrm{F} 116 & 178.2(7) \\ \mathrm{C} 29-\mathrm{C} 28-\mathrm{H} 28 & 120.1 & \mathrm{~F} 111-\mathrm{P} 111-\mathrm{F} 115 & 91.7(7) \\ \mathrm{C} 27-\mathrm{C} 28-\mathrm{H} 28 & 120.1 & \mathrm{~F} 113-\mathrm{P} 111-\mathrm{F} 115 & 89.7(5) \\ \mathrm{C} 28-\mathrm{C} 29-\mathrm{C} 30 & 118.4(4) & \mathrm{F} 114-\mathrm{P} 111-\mathrm{F} 115 & 90.9(5) \\ \mathrm{C} 28-\mathrm{C} 29-\mathrm{H} 29 & 120.8 & \mathrm{~F} 116-\mathrm{P} 111-\mathrm{F} 115 & 89.2(6) \\ \mathrm{C} 30-\mathrm{C} 29-\mathrm{H} 29 & 120.8 & \mathrm{~F} 111-\mathrm{P} 111-\mathrm{F} 112 & 89.6(10) \\ \mathrm{N} 22-\mathrm{C} 30-\mathrm{C} 29 & 123.4(4) & \mathrm{F} 113-\mathrm{P} 111-\mathrm{F} 112 & 90.0(9) \\ \mathrm{N} 22-\mathrm{C} 30-\mathrm{H} 30 & 118.3 & \mathrm{~F} 114-\mathrm{P} 111-\mathrm{F} 112 & 89.0(9) \\ \mathrm{C} 29-\mathrm{C} 30-\mathrm{H} 30 & 118.3 & \mathrm{~F} 116-\mathrm{P} 111-\mathrm{F} 112 & 178.4(10) \\ \mathrm{C} 21-\mathrm{N} 21-\mathrm{C} 25 & 118.4(4) & \mathrm{F} 115-\mathrm{P} 111-\mathrm{F} 112 & \\ \mathrm{C} 21-\mathrm{N} 21-\mathrm{R} 121 & 126.6(3) & & \end{array}$

